\title{
microRNA-199a-3p inhibits hepatic apoptosis and hepatocarcinogenesis by targeting PDCD4
}

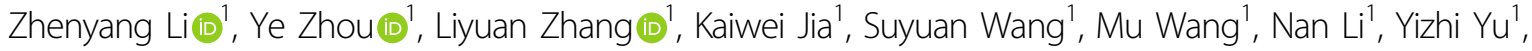 \\ Xuetao Cao (1) ${ }^{1}$ and Jin Hou (iD
}

\begin{abstract}
Hepatic apoptosis and the initiated liver inflammation play the initial roles in inflammation-induced hepatocarcinogenesis. Molecular mechanisms underlying the regulation of hepatocyte apoptosis and their roles in hepatocarcinogenesis have attracted much attention. A set of microRNAs (miRNAs) have been determined to be dysregulated in hepatocellular carcinoma (HCC) and participated in cancer progression, however, the roles of these dysregulated miRNAs in carcinogenesis are still poorly understood. We previously analyzed the dysregulated miRNAs in HCC using high-throughput sequencing, and found that miR-199a/b-3p was abundantly expressed in human normal liver while markedly decreased in HCC, which promotes HCC progression. Whether miR-199a/b-3p participates in HCC carcinogenesis is still unknown up to now. Hence, we focused on the role and mechanism of miR-199a/b-3p in hepatocarcinogenesis in this study. Hepatic miR-199a/b-3p was determined to be expressed by miR-199a-2 gene in mice, and we constructed miR-199a-2 knockout and hepatocyte-specific miR-199a-2 knockout mice.

Diethylnitrosamine (DEN)-induced hepatocarcinogenesis were markedly increased by hepatocyte-specific miR-199a$3 p$ knockout, which is mediated by the enhanced hepatocyte apoptosis and hepatic injury by DEN administration. In acetaminophen (APAP)-induced acute hepatic injury model, hepatocyte-specific miR-199a-3p knockout also aggravated hepatic apoptosis. By proteomic screening and reporter gene validation, we identified and verified that hepatic programed cell death 4 (PDCD4), which promotes apoptosis, was directly targeted by miR-199a-3p.

Furthermore, we confirmed that miR-199a-3p-suppressed hepatocyte apoptosis and hepatic injury by targeting and suppressing PDCD4. Thus, hepatic miR-199a-3p inhibits hepatocyte apoptosis and hepatocarcinogenesis, and decreased miR-199a-3p in hepatocytes may aggravate hepatic injury and HCC development.
\end{abstract}

\section{Introduction}

Hepatocellular carcinoma (HCC) is a kind of lethal malignant primary liver cancer and ranks fourth on the cancer-related death list and sixth in the terms of incident cases worldwide, which has a low 5 -year survival rate ${ }^{1,2}$. Liver diseases, such as chronic hepatitis caused by hepatitis $\mathrm{B}$ or $\mathrm{C}$ virus (HBV or $\mathrm{HCV}$ ), alcoholic liver disease and non-alcoholic fatty liver disease, increase the risk of hepatocellular carcinoma development ${ }^{3}$. Generally, HCC

\footnotetext{
Correspondence: Xuetao Cao (caoxt@immunol.org) or

Jin Hou (houjin@immunol.org)

${ }^{1}$ National Key Laboratory of Medical Immunology \& Institute of Immunology, Second Military Medical University, 200433 Shanghai, China

These authors contributed equally: Zhenyang Li, Ye Zhou, Liyuan Zhang
}

carcinogenesis is caused by the repeated cycle of hepatic injury, induced liver inflammation, and compensatory hepatocyte proliferation ${ }^{4}$. The liver injury plays a critical role in this vicious cycle, and death of hepatocytes is able to release damage-associated molecular patterns (DAMPs), which activates immune cells in the liver, recruits circulatory inflammatory cells, and initiates liver inflammation. The induced liver inflammation stimulates compensatory hepatocyte proliferation, while hepatic inflammatory responses also worsen hepatic damage and aggravate liver inflammation. Sustained hepatic injury induces the chronic liver inflammation and repeated compensatory hepatocyte proliferation, which eventually lead to hepatocarcinogenesis ${ }^{5,6}$. The underlying regulatory 
mechanisms of hepatocarcinogenesis have attracted much attention but remain largely unknown, which needs further investigation.

Cell death plays pivotal roles in the initiation of hepatocarcinogenesis. Apoptosis, necroptosis, pyroptosis, and ferroptosis are the intensively explored types of cell death during tissue damage, and different types of hepatocyte death eventually lead to different types of liver cancer ${ }^{7}$. For instance, the necroptosis of hepatocytes incubates an environment, which determines intrahepatic cholangiocarcinoma (ICC) outgrowth. However, if hepatocytes are in the environment created by apoptotic hepatocytes, they are inclined to become $\mathrm{HCC}^{8}$. Hepatocyte-specific deletion of the IKB kinase (IKK) subunit NEMO/IKK $\gamma$ sensitizes hepatocyte apoptosis by NF- $\mathrm{KB}$ inhibition, which spontaneously forms mouse HCC in 12 months $^{9}$. Similarly, mice lacking the anti-apoptotic myeloid cell leukemia-1 (Mcl-1) in hepatocytes have severe liver damage caused by spontaneous apoptosis, and tumor formation is observed in over $50 \%$ of mice in 8 months ${ }^{10}$. Besides, mice with combined knockout of receptorinteracting serine/threonine-protein kinase 1 (RIPK1) and Rel-like domain-containing protein A (RelA) in hepatocytes show increased hepatocyte apoptosis and develop spontaneous $\mathrm{HCC}^{11}$. Furthermore, other proteins regulating hepatocyte apoptosis, such as TGF- $\beta$-activated kinase 1(TAK1), TNF receptor-associated factor 2 (TRAF2), and ІкB kinase subunit beta (IKK $\beta$ ), also participate in hepatocarcinogenesis ${ }^{12,13}$. Therefore, hepatocyte apoptosis is critical in the initiation of hepatocarcinogenesis, and the molecular regulatory mechanisms of hepatocyte apoptosis and their roles in hepatocarcinogenesis have raised widespread concerns.

MicroRNAs (miRNAs) are a class of single-strand RNA with approximately 22 nucleotides in length. The functions of miRNAs have been widely investigated, and they are determined to play critical roles in cancer progression, especially in HCC. A set of miRNAs, such as miR-330-5p, miR-520a, and miR-483-3p, have been suggested to participate in cancer progression, by modulating proliferation, apoptosis, migration, and invasion ${ }^{14-16}$. However, the roles of miRNAs in hepatocarcinogenesis, especially determined in vivo, are still poorly investigated. Mice with miR-122 knockout spontaneously develop steatohepatitis, fibrosis, and eventually HCC in 11 months ${ }^{17}$. Yet, it remains unknown whether miRNAs can regulate hepatocyte apoptosis and the related hepatocarcinogenesis in vivo up to now.

Previously, we carried out an in-depth analysis of the miRNomes in human normal liver tissues and HCC tissues, and found that the liver-enriched miRNA miR-199a/b-3p was dramatically decreased in HCC, which promotes HCC progression both in vitro and in vivo ${ }^{18}$. However, the potential roles of miR-199a/b-3p in hepatocarcinogenesis is still unknown. Hence, we intend to construct hepatocytespecific miR-199a/b-3p knockout mice, and explore the role and underlying mechanism of miR-199a/b-3p in hepatocarcinogenesis in this study.

\section{Results \\ Construction of miR-199a-3p knockout mice}

We previously analyzed the dysregulated miRNAs in HCC using high-throughput sequencing, and found that miR-199a/b-3p was abundantly expressed in human normal liver while markedly decreased in HCC, which promotes HCC progression ${ }^{18}$. In order to elucidate the roles of miR-199a/b-3p in hepatocarcinogenesis, we intended to knock out its expression in mouse liver. As miR-199a/b-3p is expressed mainly by miR-199a-2 gene (Chr 1) in human liver, we first examined the expression of miR-199a gene in mouse liver. miR-199a-3p was found to be abundantly expressed in mouse liver while the miR199a-5p strand was nearly not expressed (Fig. 1a), which is similar to those in human liver. There are two gene loci of miR-199a, miR-199a-1 and miR-199a-2, which may both express miR-199a-3p. We then constructed the miR199a-1 or miR-199a-2 conventional knockout mice, respectively, to determine which one is mature miR-199a3p primarily from (Supplementary Fig. S1A, B). These generated knockout mice are fertile and seem normal. Among them, mature miR-199a-3p expression was not influenced by knockout of miR-199a-1 gene (Fig. 1b), while knockout of $m i R-199 a-2$ gene deleted the expression of miR-199a-3p in the liver (Fig. 1c). Therefore, we determined that the mature miR-199a-3p was mainly expressed from miR-199a-2 gene in mouse liver.

In order to investigate the role of miR-199a-3p exclusively in hepatocytes and hepatocarcinogenesis, we then constructed the miR-199a-2 floxed mice and crossed them with Alb-cre mice to generate hepatocyte-specific miR-199a-2 knockout mice (Supplementary Fig. S1, C). There is no obvious difference in body weight and size between miR-199a-2 $2^{f f f}$ and miR-199a-2 $2^{\text {hep- } / \text { - }}$ mice, and they are both fertile. In 24-week-old miR-199a-2fff and miR-199a- $2^{\text {hep-/- }}$ mice, serum ALT, AST, and GGT levels are similar, suggesting no spontaneous liver injury by hepatic miR-199a-2 knockout (Supplementary Fig. S1D). The livers of $m i R-199 a-2^{f / f}$ and $m i R-199 a-2^{h e p-/}$ mice were examined by HE staining, and no abnormal hepatic structure was found in miR-199a-2 $2^{\text {hep-/ }}$ mice (Supplementary Fig. S1E). Altogether, no obvious spontaneous hepatic disease is induced in miR-199a- $2^{\text {hep-/ }}$ mice. In miR-199a-2 $2^{\text {hep-/ }}$ mice, miR-199a-3p expression was markedly decreased in the liver tissue, while abolished in the isolated hepatocytes (Fig. 1d). Thus, we constructed the genetic mouse model of hepatic miR-199a-3p knockout, so as to investigate the role of miR-199a-3p in hepatocarcinogenesis. 
A

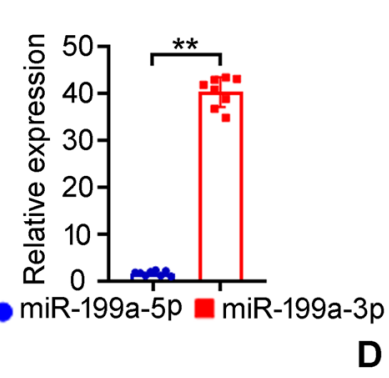

C

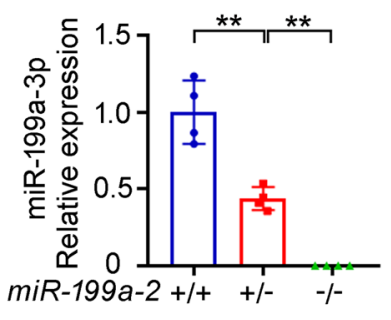

B
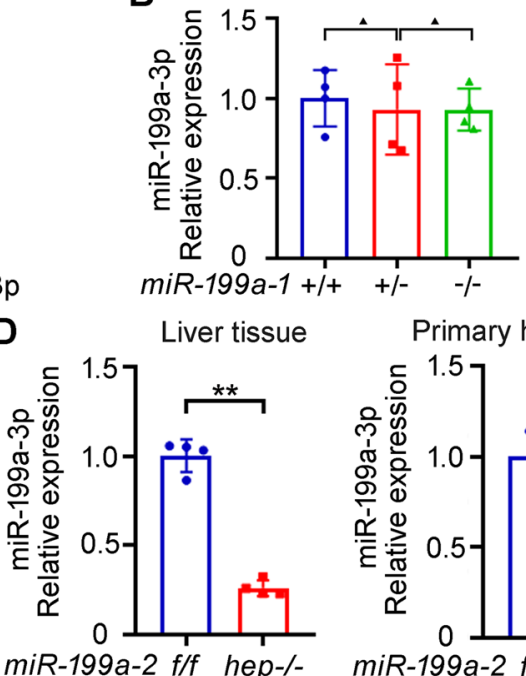

Primary hepatocyte

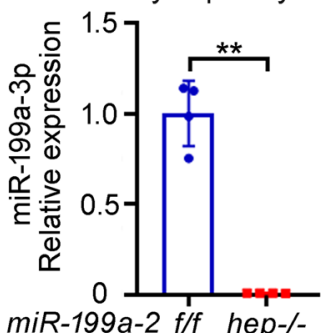

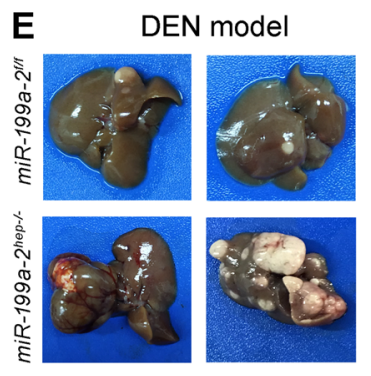

$\mathbf{F}$

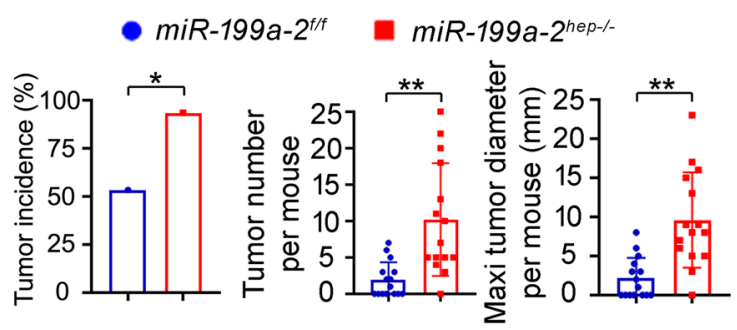

G DEN plus $\mathrm{CCl} 4$ model $\mathbf{H}$
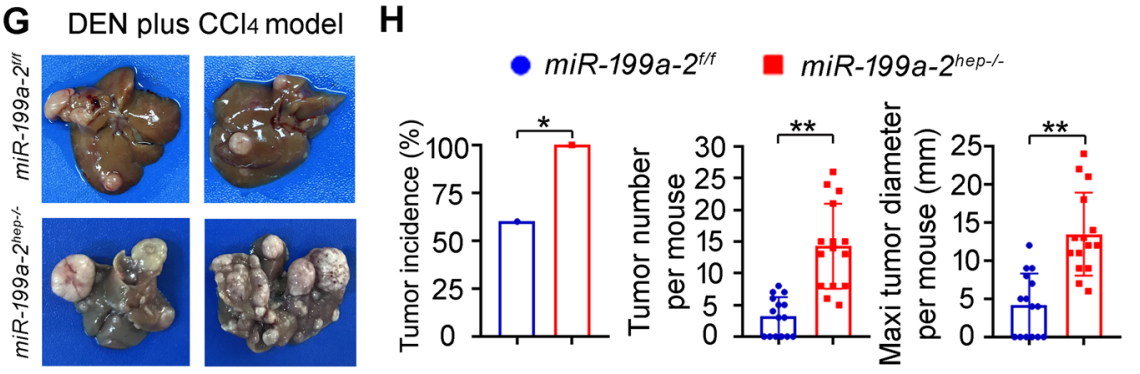

Fig. 1 Hepatocyte-specific miR-199a-2 knockout promotes hepatocarcinogenesis. a The expression of miR-199a-5p and miR-199a-3p was detected by qRT-PCR in mouse liver tissues $(n=8)$. $\mathbf{b}$ The expression of miR-199a-3p was detected by qRT-PCR from liver tissues of miR-199a- $1^{+/+}$, miR-199a-1 ${ }^{+/-}$and miR-199a-1/- mice $(n=4)$. c The expression of miR-199a-3p was detected by qRT-PCR from liver tissues of miR-199a-2 ${ }^{+/+}$, miR$199 a-2^{+/-}$and miR-199a- $2^{-1-}$ mice $(n=4)$. $\mathbf{d}$ The expression of miR-199a-3p was detected by qRT-PCR from liver tissues and primary hepatocytes of miR-199a- $2^{f / f}$ and miR-199a-2 $2^{\text {hep- }}$ mice $(n=4)$. e Two-week-old miR-199a-2/f and miR-199a-2 hep-\% male mice were intraperitoneally injected with a single dose of DEN ( $25 \mathrm{mg} / \mathrm{kg}$ ) and sacrificed 8 months later. The livers were dissected and photographed. $\mathbf{f}$ Tumor incidence (chi-square test), tumor number per mouse (unpaired $t$-test), and maximal tumor diameter per mouse (unpaired $t$-test) of the DEN-induced HCC in miR-199a-2/f and miR$199 a-2^{\text {hep- } /-}$ mice were shown, respectively, as indicated $(n=15)$. g Two-week-old miR-199a- $2^{f / f}$ and miR-199a-2 $2^{\text {hep- } /-}$ male mice were intraperitoneally injected with DEN and then $\mathrm{CCl}_{4}$. The livers with DEN plus $\mathrm{CCl}_{4}$-induced $\mathrm{HCC}$ were dissected and photographed. $\mathbf{h}$ Tumor incidence (chi-square test), tumor number per mouse (unpaired $t$-test), and maximal tumor diameter per mouse (unpaired $t$-test) of the DEN plus CCl 4 -induced HCC in miR-199a$2^{f / f}$ and miR-199a-2 $2^{\text {hep- } /}$ mice were shown, respectively, as indicated $(n=15)$. Data are shown as mean \pm SD or typical photographs of one representative experiment. Similar results were obtained in three independent experiments. $\boldsymbol{\Lambda}_{P}>0.05 ;{ }^{*} P<0.05 ;{ }^{* *} P<0.01$.

\section{Hepatocyte-specific miR-199a-2 knockout promotes hepatocarcinogenesis}

To study the role of miR-199a-3p in hepatocarcinogenesis, the DEN-induced hepatocarcinogenesis model and DEN plus $\mathrm{CCl}_{4}$ model were applied (Supplementary
Fig. S1F $)^{19,20}$. In the DEN model, mice were intraperitoneally injected with DEN 14 days post birth, and were sacrificed after 8 months. In contrast to miR-199a- $2^{f / f}$ mice, the induced hepatocarcinogenesis was markedly enhanced in miR-199a-2 $2^{\text {hep-/- }}$ mice (Fig. 1e), including 
increased tumor incidence, number, and maximal diameter (Fig. 1f). Similarly, in the DEN plus $\mathrm{CCl}_{4}$ model, hepatocytespecific miR-199a-3p knockout also markedly promoted hepatocarcinogenesis, including the increased tumor incidence, number, and maximal diameter (Fig. 1g, h). Hence, we concluded that hepatocyte-specific miR-199a-3p knockout remarkably promoted hepatocarcinogenesis in vivo.

\section{Hepatic miR-199a-2 knockout promotes hepatocyte apoptosis and hepatic injury}

To illustrate the corresponding mechanism responsible for hepatic miR-199a-3p knockout-promoted hepato-

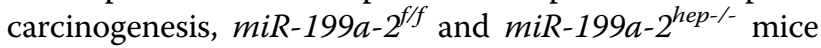
were injected with high-dose DEN to induce acute hepatic injury and liver inflammation. After DEN challenge, ballooning degeneration and coagulative necrosis were observed in the peri-central hepatocytes, which were more severe in $m i R-199 a-2^{\text {hep-/ }}$ mice than those in miR$199 a-2^{f / f}$ mice (Fig. 2a). Besides, the level of serum ALT and AST post DEN injection in miR-199a-2 $2^{\text {hep-/ }}$ mice were also significantly higher than those in $m i R-199 a-2^{f / f}$ mice (Fig. 2b). Correspondingly, TUNEL staining and cleaved caspase-3 IHC staining determined that hepatocyte apoptosis in miR-199a-2 $2^{\text {hep-/- }}$ mice was significantly more severe than that in $m i R-199 a-2^{f / f}$ mice, which was confirmed by the increased cleaved caspase- 3 in the liver of miR-199a-2 $2^{\text {hep-/ }}$ mice (Fig. 2c, d). These data suggest that hepatocyte-specific miR-199a-3p knockout promoted the hepatocyte apoptosis and hepatic injury following DEN injection.

The infiltration of inflammatory cells and compensatory proliferation of hepatocytes following acute DEN injection were also examined in the liver of $m i R-199 a-2^{\text {hep-/- }}$ mice, and they were only slightly increased as compared to those in $m i R-199 a-2^{f / f}$ mice (Fig. 2e and Supplementary Fig. S2A). Furthermore, the damage of DEN, determined by the hepatocyte DNA damage, showed no significant difference in the livers between $m i R-199 a-2^{f / f}$ and $m i R$ 199a-2 $2^{\text {hep-/ }}$ mice (Fig. 2f). Thus, the increased hepatic injury and hepatocyte apoptosis in the liver of miR-199a$2^{\text {hep-/ }}$ mice may be mediated by the direct enhancement of apoptosis under miR-199a-3p knockout.

To further determine the role of miR-199a-3p in hepatocyte apoptosis and hepatic injury, we applied the hepatic injury mouse model using acetaminophen (APAP), an anti-pyretic drug causing liver injury or even hepatic failure by misuse ${ }^{21}$. In the APAP-challenged $m i R$ $199 a-2^{f / f}$ and miR-199a-2 $2^{\text {hep-/ }}$ mice, serum ALT and AST indicating liver injury were significantly increased by hepatocyte-specific miR-199a-3p knockout (Fig. 3a). Hepatic injury around the central vein were also much heavier in the liver of in miR-199a-2 $2^{\text {hep-/ }}$ mice than that in $m i R-199 a-2^{f / f}$ mice (Fig. 3b). Besides, TUNEL and cleaved caspase-3 staining in the liver sections showed significantly more apoptotic peri-central hepatocytes in miR-199a-2 $2^{\text {hep-/ }}$ mice, which was confirmed by western blot (Fig. 3c, d). The infiltrated inflammatory cells were increased in the damaged hepatic region of miR-199a$2^{\text {hep-/ }}$ mice, and the compensatory hepatocyte proliferation was also increased (Fig. 3e and Supplementary Fig. S2B). Additionally, the direct damage of APAP, which is the induced reactive oxygen species (ROS) in hepatocytes, was similar in the liver between $m i R-199 a-2^{f / f}$ and $m i R$ $199 a-2^{\text {hep-/ }}$ mice (Fig. 3f). Together, all these data suggest that hepatocyte apoptosis were enhanced by miR-199a-3p knockout, which may be responsible for the increased hepatocarcinogenesis.

\section{Hepatic miR-199a-3p knockout promotes apoptosis in vitro}

In order to confirm the pro-apoptotic function of miR199a-3p knockout in hepatocytes, we used the APAPinduced apoptosis model in vitro, and the miR-199a-2 knockout hepatocyte cell lines were constructed and verified (Fig. 4a). As compared to those in control cell lines, miR-199a-2 knockout human hepatocyte HL-7702 cells and mouse hepatocyte BNL CL.2 cells exhibited markedly increased apoptosis, respectively, following APAP administration (Fig. 4b, c). The APAP-induced cleaved caspase-3 was also significantly enhanced by miR199a-3p knockout in these hepatocyte cell lines (Fig. 4d). Moreover, the primary hepatocytes were isolated from $m i R-199 a-2^{f f f}$ and miR-199a-2 $2^{\text {hep- }}$ mice, respectively, and the APAP-induced cleaved caspase- 3 was confirmed to be significantly promoted by miR-199a-2 knockout (Fig. 4e). Thus, miR-199a-3p knockout promotes apoptosis in both primary hepatocytes and hepatocyte cell lines in vitro.

\section{Hepatic miR-199a-3p knockout promotes apoptosis through the mitochondrial pathway}

We next intended to figure out the hepatic miR-199a-3p knockout-promoted apoptosis was through death receptor pathway or mitochondrial pathway. The cleavage and activation of caspase-8, characteristic of death receptor apoptosis pathway, were examined in both DEN and APAP-induced hepatocyte apoptosis. Using death receptor pathway activator TNF- $\alpha$ plus $\mathrm{CHX}$ in vitro and LPS plus D-gal in vivo as the positive control ${ }^{22-24}$, we did not find the caspase- 8 cleavage and death receptor pathway activation in hepatocytes upon DEN or APAP administration (Supplementary Fig. S3A-D). Thus, death receptor apoptosis pathway is not activated in DEN or APAPinduced hepatocyte apoptosis, and miR-199a-3p is less likely to inhibit hepatocyte apoptosis through suppressing death receptor pathway.

In mitochondrial apoptosis pathway, the classical proapoptotic BAK and BAX and anti-apoptotic BCL-2 and 


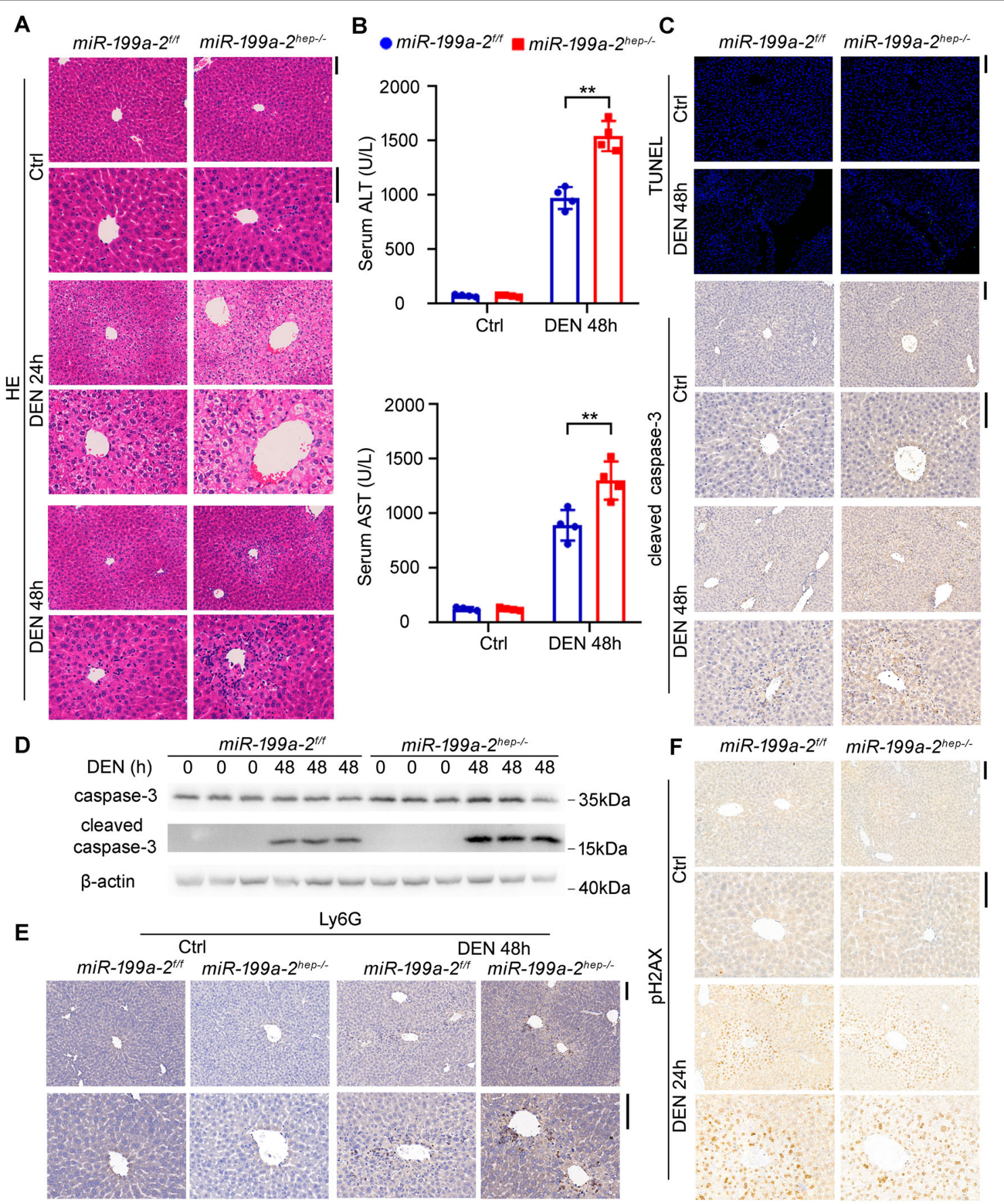

Fig. 2 Hepatocyte-specific miR-199a-3p knockout promotes DEN-induced hepatocyte apoptosis and hepatic injury. Eight-week-old miR199a-2/f and miR-199a-2 hep-/ male mice were intraperitoneally injected with DEN (100 mg/kg). Livers and serum were collected at the indicated time points. HE staining of liver sections (a), Serum ALT and ALT $(n=4)(\mathbf{b})$, TUNEL and cleaved caspase-3 IHC staining (c), cleaved caspase-3 blot (d), Ly6G staining for inflammatory cell infiltration (e), and pH2AX staining for DNA damage (f) were measured in the indicated time points post DEN challenge. Data are shown as mean \pm SD or typical photographs of one representative experiment. Similar results were obtained in three independent experiments. ${ }^{* *} P<0.01$. Scale bars, $100 \mu \mathrm{m}$.

BCL- $\mathrm{X}_{\mathrm{L}}$ were examined in DEN or APAP-induced hepatocyte apoptosis. In APAP-induced hepatocyte apoptosis, the levels of these four proteins were not changed both in vitro and in vivo (Supplementary Fig. S3E, F, G). In
DEN-induced hepatocyte apoptosis, the levels of both BAX and BCL- $\mathrm{X}_{\mathrm{L}}$ were increased upon DEN injection (Supplementary Fig. S3H). In miR-199a-2 $2^{\text {hep } / \text { - }}$ mice, the level of hepatic BCL- $\mathrm{X}_{\mathrm{L}}$ was significantly decreased as 

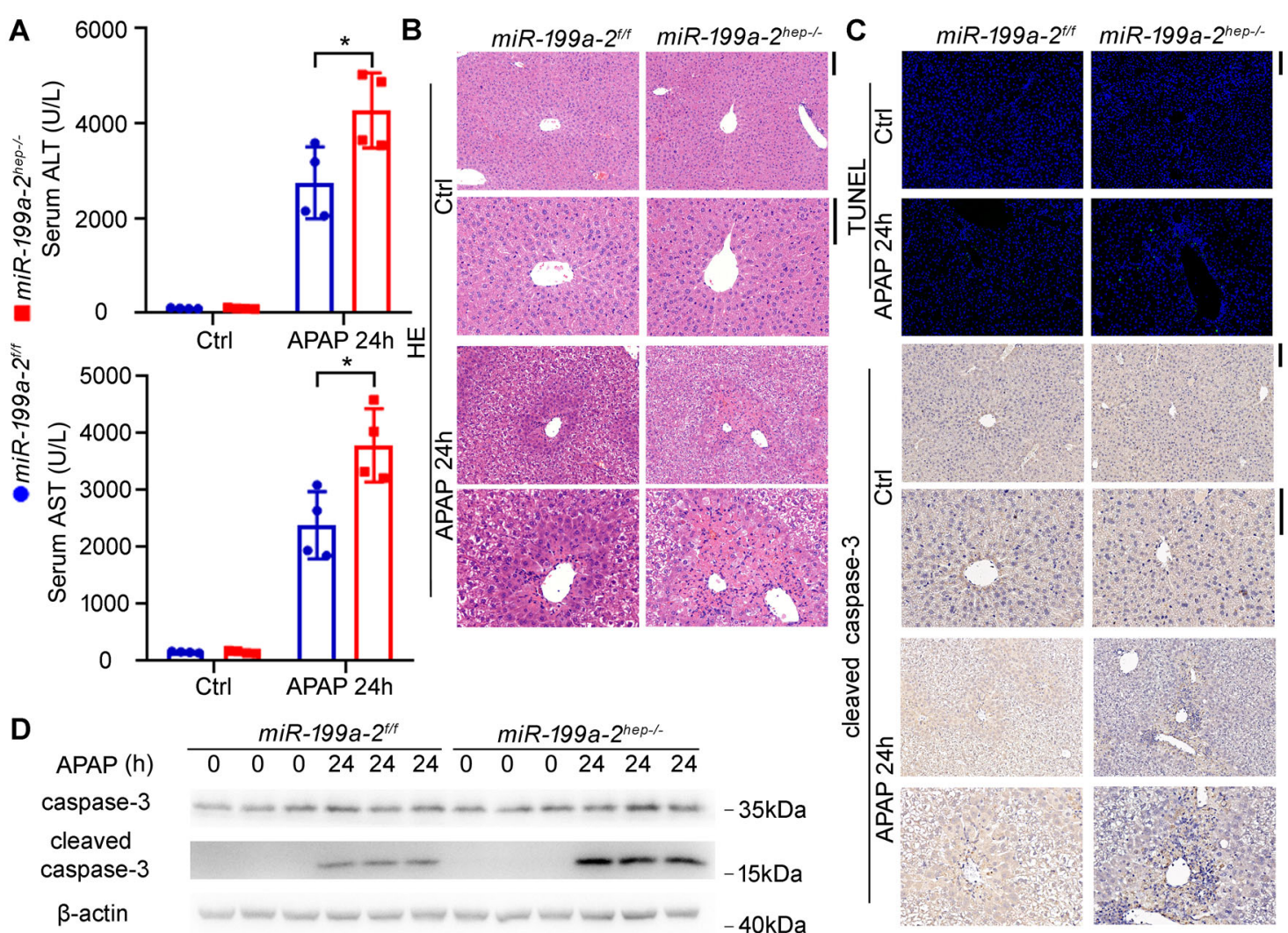

E

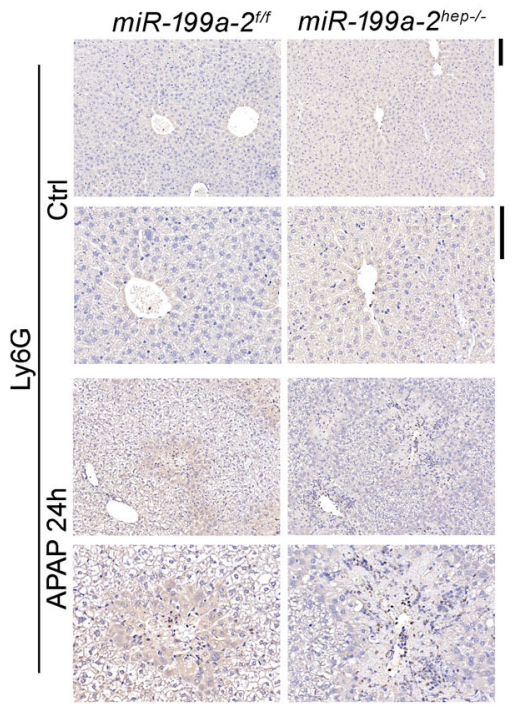

$\mathbf{F}$
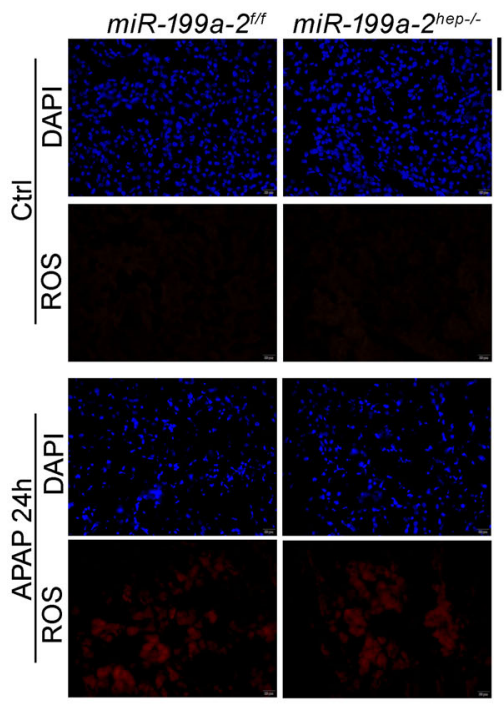

Fig. 3 Hepatocyte-specific miR-199a-3p knockout promotes APAP-induced hepatocyte apoptosis and hepatic injury. Eight-week-old miR$199 a-2^{\text {ff/f }}$ and miR-199a-2 $2^{\text {hep } /-}$ male mice were fasted for $16 \mathrm{~h}$ and then intraperitoneally injected with APAP (400 mg/kg). Livers and serum were obtained at the indicated time points. Serum ALT and ALT $(n=4)(\mathbf{a})$, HE staining $(\mathbf{b})$, TUNEL and cleaved caspase-3 IHC staining (c), cleaved caspase3 blot (d), Ly6G staining for inflammatory cell infiltration (e), and DHE staining for ROS (f) were measured in the indicated time points post APAP injection. Data are shown as mean \pm SD or typical photographs of one representative experiment. Similar results were obtained in three independent experiments. ${ }^{*} P<0.05$. Scale bars, $100 \mu \mathrm{m}$.

compared to that in $m i R-199 a-2^{f / f}$ mice, and similar results were obtained in hepatocyte cell lines (Supplementary Fig. S3E-H). These data suggest that miR-199a-2 knockout may inhibit BCL- $\mathrm{X}_{\mathrm{L}}$ expression, so as to enhance the activation of hepatic mitochondrial apoptosis pathway. 


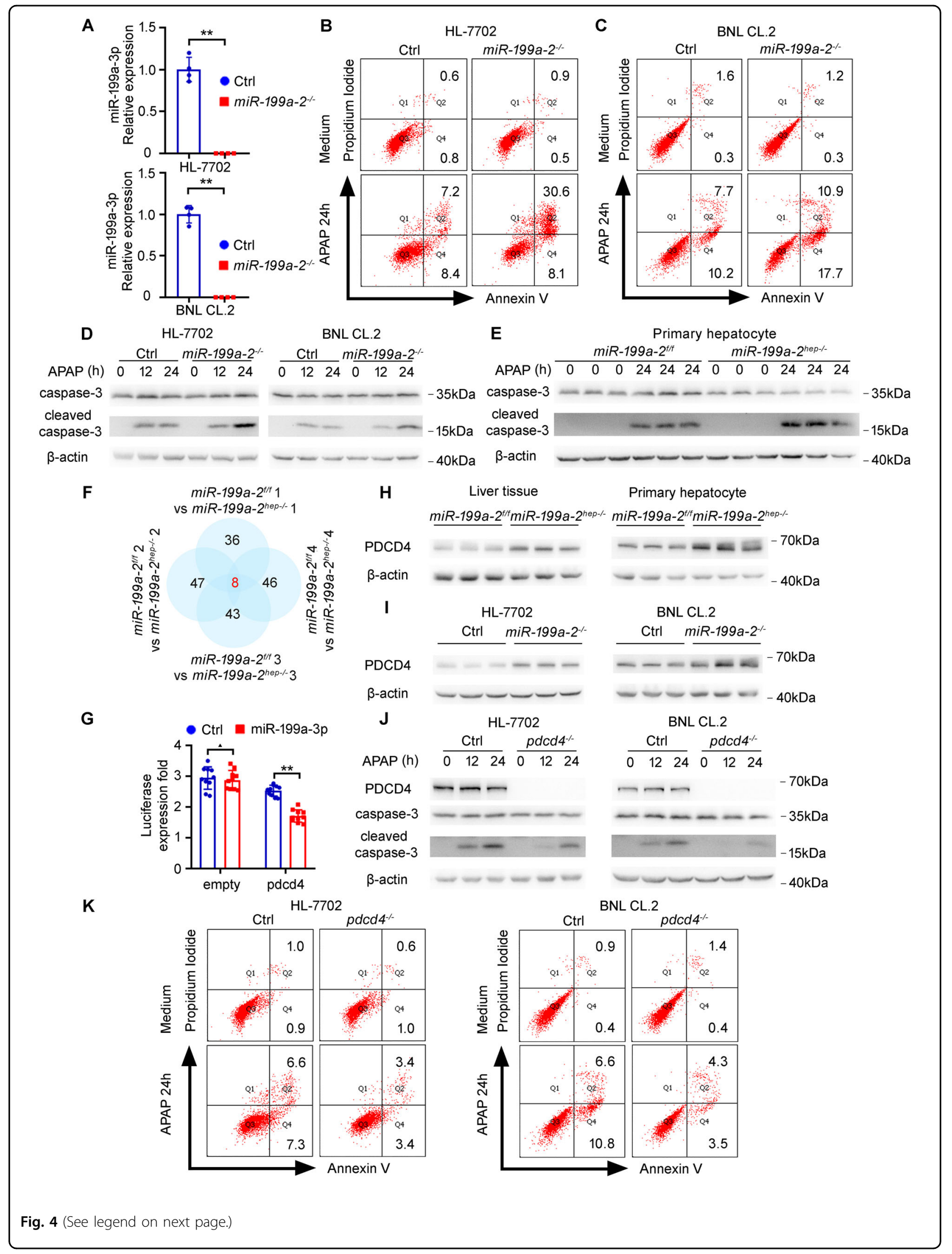


(see figure on previous page)

Fig. 4 Loss of miR-199a-3p promotes hepatocyte apoptosis by directly targeting pro-apoptotic PDCD4. a The expression of miR-199a-3p was detected by qRT-PCR in control and miR-199a-2-- cell lines. b-e Control or miR-199a-2 knockout hepatocyte cell lines were treated by APAP (10 mM) for $24 \mathrm{~h}$ as indicated. Cell apoptosis was analyzed by flow cytometry $(\mathbf{b}, \mathbf{c})$, and cleaved caspase-3 was examined by Western blot (d, e). $\mathbf{f}$ The comparative differential proteins in liver tissues from miR-199a- $2^{f / f}$ and miR-199a-2 hep-1/ mice were shown as indicated, which were from four biological repeats. $\mathbf{g}$ The indicated Firefly luciferase reporter plasmids and miR-199a-3p or control mimics were transfected into the HEK $293 \mathrm{~T}$ cells. After $24 \mathrm{~h}$, Firefly luciferase activity was measured and normalized by Renilla luciferase activity $(n=10)$. $\mathbf{h}$ Protein level of PDCD4 in liver tissues or primary hepatocytes from miR-199a-2/f and miR-199a-2hep-/ mice was measured by Western blot. i Protein level of PDCD4 in control or miR-199a-2 knockout hepatocyte cell lines was measured by western blot. $\mathbf{j}, \mathbf{k}$ Control and pdcd4 knockout hepatocyte cell lines were treated with APAP for the indicated time points. PDCD4, caspase-3, and cleaved caspase-3 were measured by Western blot (j), and cell apoptosis was measured by flow cytometry (k). Data are shown as mean \pm SD or typical photographs of one representative experiment. Similar results were obtained in three independent experiments. $\boldsymbol{\Delta}_{P}>0.05 ;{ }^{*} P<0.01$.

Furthermore, we also examined the release of cytochrome $\mathrm{C}$ into the cytosol, which is the characteristic of mitochondrial apoptosis pathway activation. In both DEN and APAP-induced release of cytochrome C, cytosol cytochrome $\mathrm{C}$ was significantly increased by hepatic $m i R$ 199a-2 knockout (Supplementary Fig. S3I-L), suggesting that miR-199a-3p knockout promotes the activation of mitochondrial apoptosis pathway in hepatocytes upon DEN or APAP administration.

\section{Hepatic PDCD4 expression is directly targeted by miR- 199a-3p}

The corresponding mechanism responsible for miR199a-3p knockout-promoted apoptosis was then investigated. As miRNAs function mainly through the inhibition of their target genes expression, we screened the differential proteins between the liver tissues from miR-199a$2^{f / f}$ and $m i R-199 a-2^{h e p-/-}$ mice using proteomic tandem mass tags (TMT) mass spectrometry (MS) analysis. Among the identified differentially expressed proteins in the liver of $m i R-199 a-2^{f / f}$ and $m i R-199 a-2^{\text {hep-l- }}$ mice, eight of them with good reproducibility were chosen for further analysis (Fig. 4f and Supplementary Fig. S4A). By sequence alignment and functional annotation, we focused on the increased PDCD4 in miR-199a-2 $2^{\text {hep- } /-}$ liver, which bears miR-199a-3p conserved target sequence and is known for its ability to promote apoptosis (Supplementary Fig. S4B) ${ }^{25,26}$. The direct targeting of PDCD4 mRNA by miR-199a-3p was validated using dualluciferase reporter assay (Fig. 4g). The mRNA level of PDCD4 was not influenced by miR-199a-3p knockout (Supplementary Fig. S4,C, D), while the protein level of PDCD4 was significantly increased by miR-199a-3p knockout in liver tissues, primary hepatocytes, and hepatocyte cell lines (Fig. 4h, i). Additionally, the proapoptotic function of PDCD4 was validated in hepatocyte cell lines, as PDCD4 knockout inhibited while PDCD4 overexpression promoted APAP-induced apoptosis (Fig. $4 \mathrm{j}, \mathrm{k}$ and Supplementary Fig. S4,E). As PDCD4 is known to repress internal ribosome entry site-mediated translation of $\mathrm{BCL}-\mathrm{X}_{\mathrm{L}}$ and suppress its expression ${ }^{25}$, the decreased BCL- $\mathrm{X}_{\mathrm{L}}$ expression was also determined above in the miR-199a-3p knockout hepatocyte cell lines and liver tissues (Supplementary Fig. S3E-H). Altogether, these data suggest that miR-199a-2 knockout may increase PDCD4 to inhibit BCL- $\mathrm{X}_{\mathrm{L}}$ expression, so as to enhance the activation of hepatic mitochondrial apoptosis pathway.

\section{miR-199a-3p suppresses hepatocyte apoptosis by targeting PDCD4}

We next examined whether miR-199a-3p-suppressed hepatocyte apoptosis was dependent on PDCD4. miR199a-3p mimics was transfected into the control and PDCD4 knockout hepatocyte cell lines, and miR-199a-3pmediated inhibition of APAP-induced cleaved caspase-3 was abolished in PDCD4 knockout cell lines (Supplementary Fig. S5A). Moreover, PDCD4 was also overexpressed in control and miR-199a-2 knockout hepatocyte cell lines, and miR-199a-3p knockoutmediated increase of cleaved caspase- 3 was abolished in PDCD4 overexpressed cell lines (Supplementary Fig. S5B). Thus, the miR-199a-3p-mediated inhibition of hepatocyte apoptosis was dependent on PDCD4 in vitro.

To analyze whether miR-199a-3p-suppressed hepatocyte apoptosis was dependent on PDCD4 in vivo, PDCD4 was overexpressed in the liver of $m i R-199 a-2^{f / f}$ and $m i R$ $199 a-2^{\text {hep- } / \text { - }}$ mice through AAV8-mediated gene delivery. miR-199a-3p knockout-mediated increase of DENinduced hepatocyte apoptosis and hepatic injury, suggested by the elevated serum ALT and AST, TUNEL and cleaved caspase- 3 staining, and cleaved caspase- 3 blot, were abolished in the PDCD4 overexpressed livers (Fig. $5 \mathrm{a}-\mathrm{c})$. Similarly, in the APAP-induced hepatic injury model, miR-199a-3p knockout-promoted hepatocyte apoptosis and hepatic injury were also abolished in the PDCD4 overexpressed livers (Fig. 6a-c). Altogether, these data determine that miR-199a-3p suppresses hepatocyte apoptosis and hepatic injury by targeting PDCD4.

\section{Discussion}

Hepatocyte death and liver injury cause the release of DAMPs and the induced liver inflammation, which 

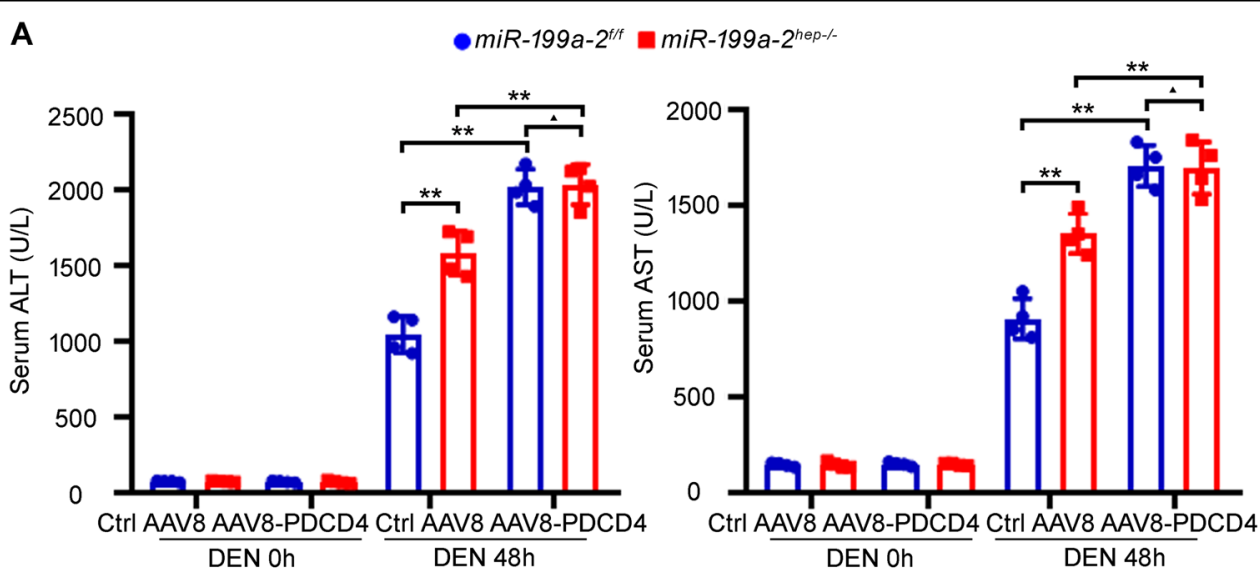

B
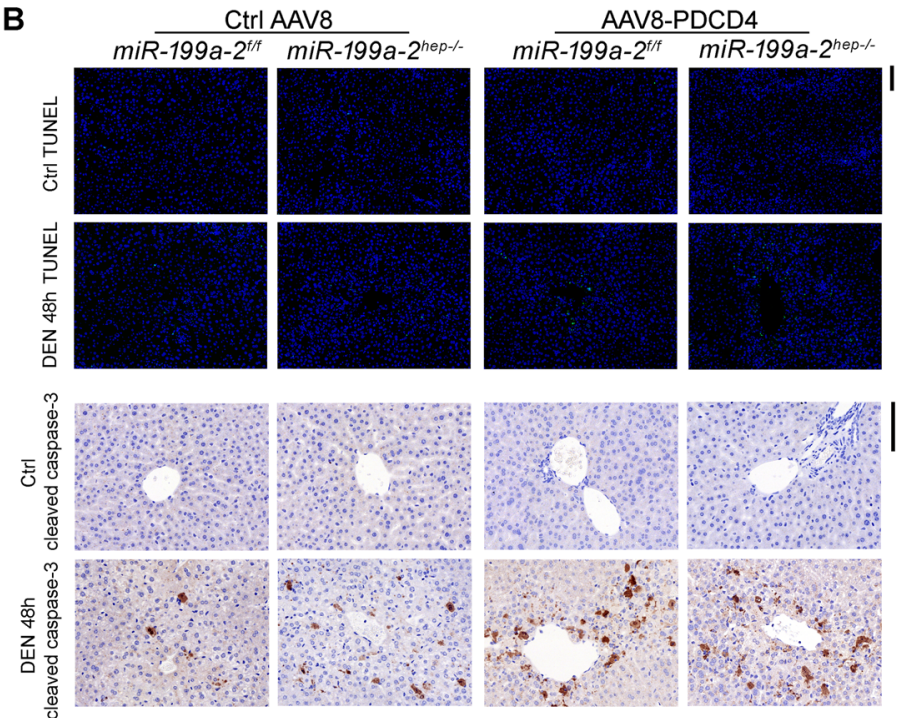

C

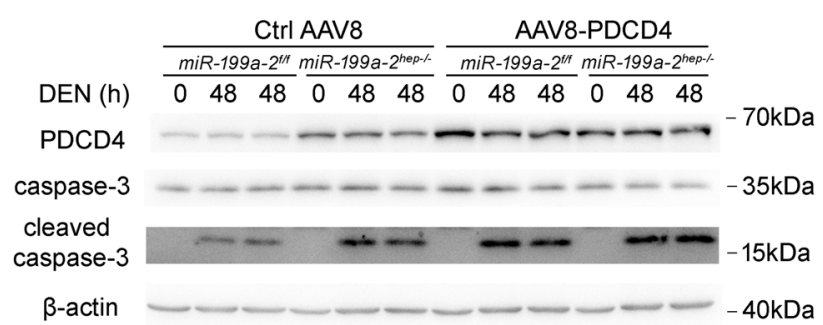

Fig. 5 miR-199a-3p inhibits DEN-induced hepatocyte apoptosis and hepatic injury by targeting PDCD4 in vivo. Control AAV8 or AAV8PDCD4 were injected into miR-199a- $2^{f / f}$ or miR-199a- $2^{\text {hep- }}$ mice through tail vein at a dose of $1 \times 10^{12} \mathrm{vg}$ per mouse, and DEN (100 mg/kg) was injected 4 weeks later. Serum ALT and AST $(n=4)(\mathbf{a})$, TUNEL and cleaved caspase-3 IHC staining $(\mathbf{b})$, and cleaved caspase-3 blot (c) were measured in the indicated time points post DEN administration. Data are shown as mean \pm SD or typical photographs of one representative experiment. Similar results were obtained in three independent experiments. $\boldsymbol{\Delta} P>0.05 ;{ }^{* *} P<0.01$.

subsequently stimulate compensatory hepatocyte proliferation and repair of liver function. However, the repeated vicious cycles of chronic injury, inflammation, and hepatocyte proliferation cause liver cirrhosis and hepatocarcinogenesis $^{27}$. During this process, hepatocyte death and liver injury play critical roles in this cycle, and hepatocyte apoptosis-initiated inflammatory circumstances are determined to induce the development of $\mathrm{HCC}^{28}$. Thus, understanding the intracellular mechanisms responsible for the control of hepatocyte apoptosis is important in future investigation of the strategies for the intervention of HCC. Based on our previous study that miR-199a-3p was abundantly expressed in the liver, we found here that hepatic miR-199a-3p was able to inhibit the hepatocyte apoptosis 


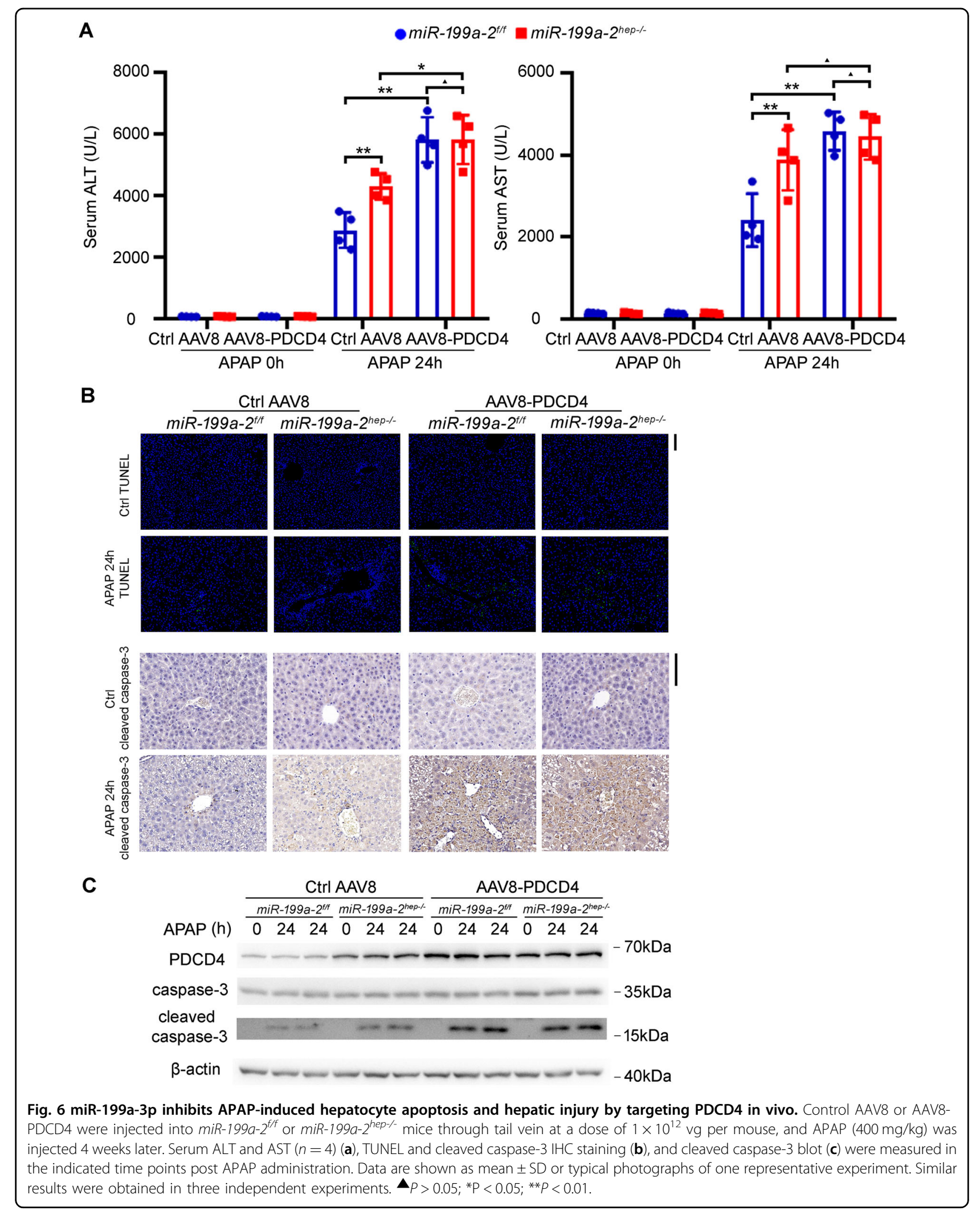


and liver injury, thus suppressing the subsequent hepatocarcinogenesis. Moreover, the apoptosis inhibition mediated by hepatic miR-199a-3p may also suggest its role in maintaining liver function during acute liver injury. Although hepatic miR-199a-2 knockout does not lead to spontaneous liver injury, it promotes hepatocyte apoptosis upon DEN injection, while other aspects such as hepatocyte DNA damage and compensatory proliferation were not influenced. Together with the pivotal role of hepatocyte apoptosis in driving HCC carcinogenesis, hepatic miR-199a-2 knockout-promoted hepatocarcinogenesis is at least in part mediated by the increased hepatocyte apoptosis upon DEN injection.

The development of HCC could be divided into two main courses, which is the first carcinogenesis and then progression. Currently, a set of miRNAs have been determined to play important roles in HCC progression, including enhanced proliferation, apoptosis inhibition, cell cycle progression, and promoted migration and invasion $^{14-16}$. On the other point, the functions and underlying mechanisms of miRNAs in hepatocarcinogenesis are still poorly understood, especially determined in vivo. Using miR-122 or miR-148a knockout mice, these two miRNAs have been implicated in hepatic lipid metabolism and the related hepatocarcinogenesis ${ }^{17,29}$. However, the roles of miRNAs in regulating hepatocyte apoptosis and the related hepatocarcinogenesis remain unknown. Here, hepatic abundant miR-199a-3p was determined to protect hepatocytes from apoptosis during liver injury, thus suppressing the related hepatocarcinogenesis, suggesting the important regulatory roles of miRNAs in modulating hepatocyte death and liver injury during hepatocarcinogenesis. Moreover, the potential regulatory roles of miRNAs in the induced hepatic inflammation and compensatory hepatocyte proliferation following liver injury during hepatocarcinogenesis are also important issues, which needs to be further investigated.

The inhibitory role of miR-199a-3p in hepatocarcinogenesis was determined using DEN and DEN plus $\mathrm{CCl}_{4}$-induced mouse hepatocarcinogenesis models. In addition, genetic models such as Tak1 $1^{\text {hep-/ }}$ or $M d r 2^{-/}$mice representing chronic liver injury, MUP-uPA transgenic mice representing continuous hepatic ER stress, and Stelic Animal Model (STAM) representing metabolic disorders-induced HCC, were also commonly used in the study of molecular regulatory mechanisms in hepatocarcinogenesis ${ }^{30-33}$. It was suggested that the HCC tissues form STAM model, induced by streptozocin and high-fat diet (HFD), were the most similar to human HCC by transcriptome analysis ${ }^{34}$. Thus, the potential roles of hepatic abundant miR-199a-3p in the STAM metabolic hepatocarcinogenesis model, including its roles in liver metabolic diseases such as NAFLD and NASH, are still unknown up to now, which will be investigated in the future.
We previously reported that miR-199a-3p was abundantly expressed in normal liver but markedly decreased in HCC tissues, and decreased miR-199a-3p promoted HCC survival and growth. In this work, we found that hepatic miR-199a-3p knockout promoted hepatocarcinogenesis, and miR-199a-3p knockout enhanced hepatocyte apoptosis. Together with these data, two important scientific questions are raised. First, why does decreased miR-199a-3p enhance apoptosis in hepatocytes while promote survival in HCC? An easy explanation may be that miR-199a-3p functions differently in different cell types by targeting different genes expression. PAK4, the target of miR-199a-3p we found in HCC, was not detected by the proteomic analysis in miR-199a-3p deficient hepatocytes in this work. However, the underlying mechanisms responsible for the different main targets of a single miRNA in different cell types still need intensive investigation, and these mechanisms may unveil the different modes of interaction among molecules between benign hepatocyte and malignant HCC. Second, as miR199a-3p expression is markedly decreased in HCC, whether miR-199a-3p is decreased in hepatocytes first and then promote hepatocyte apoptosis and liver injury, or it is decreased only in established HCC to promote HCC survival and progression? The answer to this question may need the identification of detailed transformation process from hepatocyte to $\mathrm{HCC}$, and examination of miR-199a-3p expression and change of function in the interim cell types between hepatocytes and HCC. Additionally, the possibility also exists that miR-199a-3p is abundantly expressed in hepatocytes and maintain their survival and liver function before HCC formation, while only decreased in established $\mathrm{HCC}$, when its function changes and decreased miR-199a-3p promotes HCC survival and progression. These two questions may raise interesting future works in this issue.

Another interesting point is that there are three gene loci to express mature miR-199-3p, including miR-199a1, miR-199a-2, and miR-199b, in the genome of both human and mouse as miRNAs are evolutionarily conserved non-coding RNAs. In the liver, we determined that miR-199-3p was mainly from miR-199a-2, because miR199-3p is not detectable when miR-199a-2 is knocked out. In some other organs such as spleen and kidney, miR-199$3 p$ is also from miR-199a-2 gene, which is identified in miR-199a-2 knockout mice (data not shown), and we presume that miR-199a-3p may work similarly to suppress apoptosis and maintain function in these organs. However, deletion of miR-199a-2 does not reduce miR-199-3p expression in lung and colon, while miR-199a-1 knockout does (Data not show), suggesting that miR-199 is expressed by different gene locus in different organs. As miR-199 was reported to be dysregulated in cancer from all these organs ${ }^{35-37}$, the 
corresponding mechanisms responsible for the dysregulation of miR-199 may be also different. Moreover, in mouse islets, miR-199a-3p is from both miR-199a-1 and miR-199a2, but not miR-199b, and the pri-miR-199a-1 is about 30\% more than pri-miR-199a-2, whose expression are regulated by glucose metabolism and calcium influx ${ }^{38}$. Thus, why miR-199-3p is expressed from different gene locus in different organs and the underlying biological significance are interesting topics, which still need further exploration.

For the roles of PDCD4 in carcinogenesis, previous reports suggested that PDCD4 knockout mice could spontaneously develop lymphoma around 85 weeks, which may be mediated by the upregulated IL- 4 and IL-10 produced by $p d c d 4^{-/-}$immune cells ${ }^{39}$. Besides, PDCD4 knockout also promoted AOM plus DSS-induced colorectal cancer by the mechanism that PDCD4 deficiency increased the macrophage-secreted IL- 6 induced by DSS and elevated the epithelial cell susceptibility to IL-6/ STAT3 pathway-mediated cell proliferation during malignant transformation ${ }^{40}$. In this work, we observed the increased level of PDCD4 in miR-199a-2 $2^{\text {hep-/- }}$ mice, which enhanced hepatocyte apoptosis induced by DEN and APAP, and the promoted hepatocarcinogenesis in $m i R$ $199 a-2^{\text {hep-/- }}$ mice induced by DEN and DEN plus $\mathrm{CCl}_{4}$. In different types of cells, the functions of PDCD4 are probably to be different, which may be mediated by the different PDCD4-regulated gene expression and signaling activation. This description may explain the probable different roles of PDCD4 in lymphocytes, macrophages, epithelium, and hepatocytes. Although we determined here that miR-199a-3p targets PDCD4 to inhibit hepatocyte apoptosis and then suppress hepatocarcinogenesis, the direct evidence of PDCD4 in hepatocarcinogenesis is still not determined in this study, and we will keep focusing on this issue in our future study.

\section{Materials and methods \\ Mice and cell lines}

miR-199a-1 conventional knockout mice were constructed by Model Animal Research Center of Nanjing University (Nanjing, China). miR-199a-2 conventional knockout mice were constructed by Beijing VIEWSOLID BIOTECH Ltd (Beijing, China). miR-199a-2fff mice were constructed by Shanghai Model Organism Center (Shanghai, China). Identification of mouse genotype was carried out by PCR analysis using genomic DNA purified from tail tissues (Supplementary Table S1) ${ }^{41}$. All animal experiments were undertaken in accordance with National Institutes of Health's Guide for the Care and Use of Laboratory Animals with the approval of the institutional research ethics committee of Second Military Medical University, Shanghai, China. HL-7702 and BNL CL.2 cell lines were obtained from cell bank of Chinese Academy of Sciences (Shanghai, China). All cell lines have been authenticated using STR profiling and tested for mycoplasma contamination by Genechem (Shanghai, China). The knockout cell lines were constructed using CRISPR/Cas9 method (Supplementary Table S2) ${ }^{42}$. HL7702 was cultured in RPMI 1640 with 10\% Fetal Bovine Serum (FBS), and BNL CL.2 was cultured in dulbecco's modified eagle medium (DMEM) with 10\% FBS and 1\% non-essential animo acid (NAA).

\section{Reagents}

Antibodies specific to caspase-3 (9662), cleaved caspase3 (9661), caspase-8 (4790), human cleaved caspase-8 (9496), mouse cleaved caspase-8 (9429), BAK (12105), BAX (2772), BCL-2 (3498), BCL- $\mathrm{X}_{\mathrm{L}}$ (2764), PDCD4 (9535), and horseradish peroxidase-coupled secondary antibodies (7074 and 7076) were from Cell Signaling Technology (Danvers, MA). Antibody specific to $\beta$-actin (A5441), DEN (N0258), DHE (D7008), CHX (239764), LPS (L3024) and MEM NAA (M7145) were from SigmaAldrich (St. Louis, MO). Antibody specific to cytochrome C (sc-13156) was from Santa Cruz Biotechnology (CA, USA). Recombinant human TNF- $\alpha$ (300-01A) and murine TNF- $\alpha$ (315-01 A) were from Pepro Tech (Rocky Hill, NJ). TUNEL assay kit (11684817910) was from Roche (Shanghai, China). $\mathrm{CCl}_{4}$ (CAS: 56-23-5, C805329) was from MAKLIN (Shanghai, China). Olive oil (CAS: 800125-0, A502795-0100), APAP (CAS: 103-90-2, A506808), and D-hanks buffer (B548148-0500) were from Sangon Biotech (Shanghai, China). Cell mitochondria isolation kit (C3601) and tissue mitochondria isolation kit (C3606) were from Beyotime (Shanghai, China). Percoll solution (17-0891-01) was from GE Healthcare Life Science (Little Chalfont, UK). Type IV collagenase (LS004140) was from Worthington Biochemical Corporation (Lakewood, NJ). Protease inhibitor cocktail (539134-1SML) and apoptosis assay kit (PF032) were from Calbiochem (Darmstadt, Germany).

\section{Establishment of hepatocarcinogenesis model}

To construct DEN-induced hepatocarcinogenesis model, the 2-week-old male mice were injected with $25 \mathrm{mg} / \mathrm{kg}$ body weight DEN intraperitoneally ${ }^{43}$. Mice were sacrificed 8 months later and tumors were analyzed. As for DEN plus $\mathrm{CCl}_{4}$ model, the 2-week-old male mice were injected with $25 \mathrm{mg} / \mathrm{kg}$ body weight DEN intraperitoneally, and 4 weeks later, mice were injected with $\mathrm{CCl}_{4}$ $(0.5 \mathrm{ml} / \mathrm{kg}$ body weight, dissolved in olive oil at a ratio of 1:3) weekly lasting for 15 weeks $^{19}$. Mice were sacrificed 8 weeks after last $\mathrm{CCl}_{4}$ injection.

\section{Establishment of acute hepatic injury model}

To constructed DEN-induced acute hepatic injury model, 8-week-old male mice were injected with DEN $(100 \mathrm{mg} / \mathrm{kg})$ intraperitoneally and mice were sacrificed 
according to the time points. As for APAP-induced acute hepatic injury model, eight-week-old male mice were fasted overnight $(16 \mathrm{~h})$ and then injected with APAP $(400 \mathrm{mg} / \mathrm{kg})$ intraperitoneally. Mice were fed immediately after injection and sacrificed as indicated. Serum ALT and AST were measured by automatic biochemical analyzer FDC-7000i (Shanghai, China).

\section{In vivo AAV8 administration}

The rAAV (serotype 8) vector expressing PDCD4 under promoter CAG was constructed as previously described ${ }^{18}$. For AAV8 administration, $1 \times 10^{12} \mathrm{vg}$ AAV8 per mouse in $200 \mu \mathrm{l}$ saline buffer was injected into the mice through tail vein. Four weeks later, the protein expression of PDCD4 was measured by Western blot.

\section{Isolation of primary hepatocytes}

Primary hepatocytes were isolated from mouse liver using two-step collagenase perfusion procedure ${ }^{44}$. Mice were anesthetized and first perfused with $45 \mathrm{ml} \mathrm{D}$-hanks buffer and then with type IV collagenase digestion $(1 \mathrm{mg} /$ $\mathrm{ml}$ ) for $5 \mathrm{~min}$ at a rate of $2 \mathrm{ml} / \mathrm{min}$ through the portal vein. After digestion, the liver was excised, minced, filtered through 70-micron membrane, spin for $5 \mathrm{~min}$ at $50 \times g$, and purified using 50\% Percoll solution. The obtained hepatocytes were resuspended in DMEM, and trypan blue exclusion assays indicated that cell viability was $>95 \%$. Primary hepatocytes were cultured in DMEM with 10\% FBS and 1\% Penicillin-Streptomycin.

\section{RNA isolation and quantitative PCR analysis}

Total RNA was extracted from mouse liver tissues or cell lines using RNAiso Plus reagent (Takara, Dalian, China) according the recommended protocol. Real-time quantitative RT-PCR (qRT-PCR) analysis was performed using LightCycler 2.0 (Roche, Switzerland) and SYBR RTPCR kit (Takara) as previously described ${ }^{18}$. The relative expression level of the individual genes was normalized to that of internal control by using $2^{-\Delta \Delta \mathrm{Ct}}$ cycle threshold method in each sample (Supplementary Table S3) ${ }^{45}$.

\section{Western blot}

Cells or tissues were lysed on ice with cell lysis buffer (Cell Signaling Technology) supplemented with protease inhibitor cocktail (Calbiochem) at a ratio of 1:200. Protein concentrations of the extracts were measured with bicinchoninic acid (BCA) assay (Pierce). Equal amount of the extracts was loaded to sodium dodecyl sulfate polyacrylamide gel electrophoresis and transferred onto nitrocellulose membrane for immunoblot analysis as described previously $y^{20}$.

\section{Histology}

For HE, IHC and TUNEL, liver tissues were fixed in $4 \%$ paraformaldehyde for $48 \mathrm{~h}$, embedded by paraffin and sliced up into $5 \mu \mathrm{m}$ thick sections, which were processed to HE, IHC, and TUNEL as kit protocols described. For reactive oxygen species (ROS) measurement, fresh liver tissues were embedded by Tissue-Tek ${ }^{\circledR}$ O.C.T compound and immediately sliced up into liver sections. The slices were incubated in DHE $(1 \mu \mathrm{M})$ for $30 \mathrm{~min}$. After washing with PBS for three times, the red fluorescence was measured by fluorescence microscope.

\section{Dual-luciferase reporter assay}

The PDCD4 luciferase reporter was made by amplifying the mouse $p d c d 4$ mRNA by PCR and cloned into the 3UTR region of pMIR-promoter-Firefly plasmids. The luciferase reporter plasmids, RL-TK-Renilla plasmids, and indicated miRNAs (final concentration $20 \mathrm{nM}$ ) were cotransfected into HEK 293T cells. After $24 \mathrm{~h}$, the activities of Firefly and Renilla luciferases were measured using the Dual-Luciferase Reporter Assay System (Promega) as previously described ${ }^{46}$. Data was normalized for transfection efficiency by dividing Firefly luciferase activity with that of Renilla luciferase.

\section{Flow cytometry}

The control and knockout cells were treated with APAP $(10 \mathrm{mM})$ for $24 \mathrm{~h}$. Cells were collected and labeled by apoptosis assay kit and subjected to flow cytometry analysis on LSRII. Data were analyzed using FACSDiva software (Becton Dickinson).

\section{Statistical analysis}

As indicated in the figure legends, sample size of the experiments depended on the assay type. There were no blind experiments for the investigators both in cells and mice experiments. The mice were randomly distributed to the experimental group. For all groups that are statistically compared, the variance within each group was similar. Data are shown as mean \pm SD from one representative of three independent experiments. Statistical comparisons between experimental groups were analyzed by unpaired Student's $t$-test or chi-square test in GraphPad Prism 8.0, and a two-tailed $P<0.05$ was taken to indicate statistical significance.

\footnotetext{
Acknowledgements

We thank Yuchao Yin, Tingting Fang, and Yan Li for technical support, and Prof. Taoyong Chen, Xingguang Liu, and Chaofeng Han for helpful discussion. This work was supported by Grants from the National 135 Major Project of China (2018ZX10302205 and 2017ZX10102032), the National Natural Science Foundation of China (91842104, 81871229, 81671564, 81701566, 81672798, 81872232),

Program of Shanghai Academic Research Leader (19XD1424900), Shanghai Sailing Project (17YF1424600), and Shanghai Chen Guang Project (18CG39).
}

Conflict of interest

The authors declare that they have no conflict of interest. 


\section{Publisher's note}

Springer Nature remains neutral with regard to jurisdictional claims in published maps and institutional affiliations.

Supplementary Information accompanies this paper at (https://doi.org/ 10.1038/s41389-020-00282-y).

Received: 20 April 2020 Revised: 21 September 2020 Accepted: 13 October 2020

Published online: 24 October 2020

\section{References}

1. Villanueva, A. Hepatocellular carcinoma. N. Engl. J. Med. 380, 1450-1462 (2019).

2. Siegel, R. L., Miller, K. D. \& Jemal, A. Cancer statistics, 2020. CA. Cancer J. Clin. 70, 7-30 (2020).

3. Forner, A., Reig, M. \& Bruix, J. Hepatocellular carcinoma. Lancet 391, 1301-1314 (2018).

4. Grivennikov, S. I., Greten, F. R. \& Karin, M. Immunity, inflammation, and cancer. Cell 140, 883-899 (2010).

5. Ringelhan, M., Pfister, D., O'Connor, T., Pikarsky, E. \& Heikenwalder, M. The immunology of hepatocellular carcinoma. Nat. Immunol. 19, 222-232 (2018).

6. Kubes, P. \& Jenne, C. Immune responses in the liver. Annu. Rev. Immunol. 36, 247-277 (2018)

7. Green, D. R. The coming decade of cell death research: five riddles. Cell 177, 1094-1107 (2019)

8. Seehawer, M. et al. Necroptosis microenvironment directs lineage commitment in liver cancer. Nature 562, 69-75 (2018).

9. Luedde, T. et al. Deletion of NEMO/IKKY in liver parenchymal cells causes steatohepatitis and hepatocellular carcinoma. Cancer Cell 11, 119-132 (2007).

10. Weber, A. et al. Hepatocyte-specific deletion of the antiapoptotic protein myeloid cell leukemia-1 triggers proliferation and hepatocarcinogenesis in mice. Hepatology 51, 1226-1236 (2010).

11. Van, T. M. et al. Kinase-independent functions of RIPK1 regulate hepatocyte survival and liver carcinogenesis. J. Clin. Invest. 127, 2662-2677 (2017).

12. Inokuchi, S. et al. Disruption of TAK1 in hepatocytes causes hepatic injury, inflammation, fibrosis, and carcinogenesis. Proc. Natl Acad. Sci. USA 107 844-849 (2010).

13. Schneider, A. T. et al. RIPK1 suppresses a TRAF2-dependent pathway to liver cancer. Cancer Cell 31, 94-109 (2017).

14. Xiao, S. et al. miR-330-5p targets SPRY2 to promote hepatocellular carcinoma progression via MAPK/ERK signaling. Oncogenesis 7, 90 (2018).

15. Huang, J. et al. An ANCCA/PRO2000-miR-520a-E2F2 regulatory loop as a driving force for the development of hepatocellular carcinoma. Oncogenesis 30, e229 (2016).

16. Pepe, F. et al. Regulation of miR-483-3p by the O-linked N-acetylglucosamine transferase links chemosensitivity to glucose metabolism in liver cancer cells. Oncogenesis 8, e328 (2017).

17. Tsai, W. C. et al. MicroRNA-122 plays a critical role in liver homeostasis and hepatocarcinogenesis. J. Clin. Invest. 122, 2884-2897 (2012).

18. Hou, J. et al. Identification of miRNomes in human liver and hepatocellular carcinoma reveals miR-199a/b-3p as therapeutic target for hepatocellular carcinoma. Cancer Cell 19, 232-243 (2011).

19. Hernandez, C. et al. HMGB1 links chronic liver injury to progenitor responses and hepatocarcinogenesis. J. Clin. Invest. 128, 2436-2451 (2018).

20. Hou, J. et al. Hepatic RIG-I predicts survival and interferon-a therapeutic response in hepatocellular carcinoma. Cancer Cell 25, 49-63 (2014).

21. Bunchorntavakul, C. \& Reddy, K. R. Acetaminophen (APAP or N-Acetyl-pAminophenol) and acute liver failure. Clin. Liver Dis. 22, 325-346 (2018).

22. Priem, D. et al. A20 protects cells from TNF-induced apoptosis through linear ubiquitin-dependent and -independent mechanisms. Cell Death Dis. 10, 692 (2019).
23. Jing, Z. T. et al. AKT activator SC79 protects hepatocytes from TNF-a-mediated apoptosis and alleviates d-Gal/LPS-induced liver injury. Am. J. Physiol. Gastrointest. Liver Physiol. 316, G387-G396 (2019).

24. Deng, Y. et al. Oridonin ameliorates lipopolysaccharide/D-galactosamineinduced acute liver injury in mice via inhibition of apoptosis. Am. J. Transl. Res. 9, 4271-4279 (2017).

25. Liwak, U. et al. Tumor suppressor PDCD4 represses internal ribosome entry site-mediated translation of antiapoptotic proteins and is regulated by $\mathrm{S} 6$ kinase 2. Mol. Cell Biol. 32, 1818-1829 (2012).

26. White, $\mathrm{K}$. et al. Endothelial apoptosis in pulmonary hypertension is controlled by a microRNA/programmed cell death 4/caspase-3 axis. Hypertension 64, 185-194 (2014).

27. Luedde, T. \& Schwabe, R. F. NF-KB in the liver-linking injury, fibrosis and hepatocellular carcinoma. Nat. Rev. Gastroenterol. Hepatol. 8, 108-118 (2011).

28. Kondylis, V. et al. NEMO prevents steatohepatitis and hepatocellular carcinoma by inhibiting RIPK1 kinase activity-mediated hepatocyte apoptosis. Cancer Cell 28, 582-598 (2015).

29. Cheng, L. et al. microRNA-148a deficiency promotes hepatic lipid metabolism and hepatocarcinogenesis in mice. Cell Death Dis. 8, e2916 (2017).

30. Yang, L. et al. Transforming growth factor- $\beta$ signaling in hepatocytes promotes hepatic fibrosis and carcinogenesis in mice with hepatocyte-specific deletion of TAK1. Gastroenterology 144, 1042-1054.e4 (2013).

31. Fuchs, C. D. et al. Colesevelam attenuates cholestatic liver and bile duct injury in $\mathrm{Mdr}^{-/-}$mice by modulating composition, signalling and excretion of faecal bile acids. Gut 67, 1683-1691 (2018).

32. Nakagawa, $\mathrm{H}$. et al. ER stress cooperates with hypernutrition to trigger TNFdependent spontaneous HCC development. Cancer Cell 26, 331-343 (2014).

33. van der Windt, D. J. et al. Neutrophil extracellular traps promote inflammation and development of hepatocellular carcinoma in nonalcoholic steatohepatitis. Hepatology 68, 1347-1360 (2018).

34. Dow, M. et al. Integrative genomic analysis of mouse and human hepatocellular carcinoma. Proc. Natl Acad. Sci. USA 115, E9879-E9888 (2018).

35. Liu, J. et al. miR-199a-3p acts as a tumor suppressor in clear cell renal cell carcinoma. Pathol. Res. Pract. 214, 806-813 (2018).

36. Jin, H. et al. Restoration of mutant K-Ras repressed miR-199b inhibits K-Ras mutant non-small cell lung cancer progression. J. Exp. Clin. Cancer Res. 38, 165 (2019).

37. Wan, D. et al. Aberrant expression of miR-199a-3p and its clinical significance in colorectal cancers. Med. Oncol. 30, 378 (2013).

38. Werneck-de-Castro, J. P., Blandino-Rosano, M., Hilfiker-Kleiner, D. \& BernalMizrachi, E. Glucose stimulates microRNA-199 expression in murine pancreatic ß-cells. J. Biol. Chem. 295, 1261-1270 (2020).

39. Hilliard, A. et al. Translational regulation of autoimmune inflammation and lymphoma genesis by programmed cell death 4. J. Immunol. 177, 8095-8102 (2006).

40. Wang, L. et al. PDCD4 deficiency aggravated colitis and colitis-associated colorectal cancer via promoting IL-6/STAT3 pathway in mice. Inflamm. Bowel Dis. 22, 1107-1118 (2016).

41. Zheng, Q., Hou, J., Zhou, Y., Li, Z. \& Cao, X. The RNA helicase DDX46 inhibits innate immunity by entrapping $\mathrm{mA}$-demethylated antiviral transcripts in the nucleus. Nat. Immunol. 18, 1094-1103 (2017).

42. Cong, L. et al. Multiplex genome engineering using CRISPR/Cas systems. Science 339, 819-823 (2013).

43. Han, Y. et al. Tumor-induced generation of splenic erythroblast-like Ter-cells promotes tumor progression. Cell 173, 634-648.e12 (2018).

44. Sun, P. et al. Caspase recruitment domain protein 6 protects against hepatic steatosis and insulin resistance by suppressing apoptosis signal-regulating kinase 1. Hepatology 68, 2212-2229 (2018).

45. Livak, K. J. \& Schmittgen, T. D. Analysis of relative gene expression data using real-time quantitative $P C R$ and the 2(-Delta Delta C(T)) Method. Methods 25, 402-408 (2001).

46. Hou, J. et al. MicroRNA-146a feedback inhibits RIG-I-dependent Type I IFN production in macrophages by targeting TRAF6, IRAK1, and IRAK2. J. Immunol. 183, 2150-2158 (2009). 